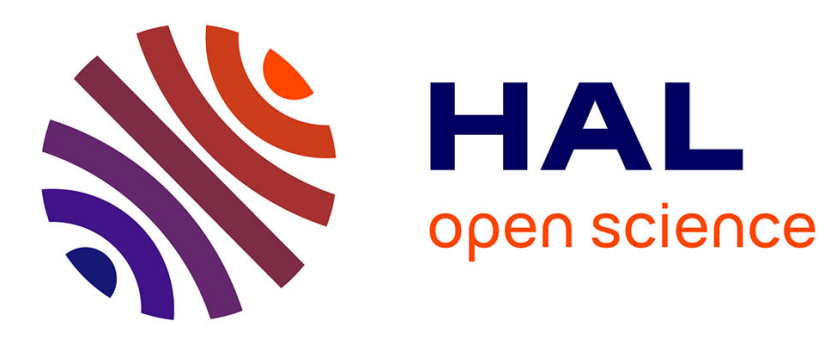

\title{
Fast and Accurate Mobile-Aided Screening System of Moderate Diabetic Retinopathy
}

Yaroub Elloumi, Manef Ben Mbarek, Rahma Boukadida, Mohamed Akil, Mohamed Hedi Bedoui

\section{- To cite this version:}

Yaroub Elloumi, Manef Ben Mbarek, Rahma Boukadida, Mohamed Akil, Mohamed Hedi Bedoui. Fast and Accurate Mobile-Aided Screening System of Moderate Diabetic Retinopathy. International Conference on Machine Vision (ICMV), Nov 2020, Rome, Italy. hal-02977506

\section{HAL Id: hal-02977506 https://hal.science/hal-02977506}

Submitted on 25 Oct 2020

HAL is a multi-disciplinary open access archive for the deposit and dissemination of scientific research documents, whether they are published or not. The documents may come from teaching and research institutions in France or abroad, or from public or private research centers.
L'archive ouverte pluridisciplinaire HAL, est destinée au dépôt et à la diffusion de documents scientifiques de niveau recherche, publiés ou non, émanant des établissements d'enseignement et de recherche français ou étrangers, des laboratoires publics ou privés. 


\title{
Fast and Accurate Mobile-Aided Screening System of Moderate Diabetic Retinopathy
}

\author{
Yaroub Elloumi ${ }^{\mathrm{a}, \mathrm{b}, \mathrm{c}}$, Manef Ben Mbarek ${ }^{\mathrm{a}}$, Rahma Boukadida ${ }^{\mathrm{a}}$, Mohamed Akil ${ }^{\mathrm{b}}$, \\ Mohamed Hedi Bedoui ${ }^{\mathrm{a}}$ \\ ${ }^{\mathrm{a}}$ Medical Technology and Image Processing Laboratory, Faculty of medicine, University of \\ Monastir, Tunisia. \\ bLIGM, Univ Gustave Eiffel, CNRS, ESIEE Paris, F-77454 Marne-la-Vallée France. \\ ${ }^{\mathrm{c}}$ ISITCom Hammam-Sousse, University of Sousse, Tunisia.
}

\begin{abstract}
The Diabetic Retinopathy (DR) is a worldwide eye disease that causes visual damages and can leads to blindness. Therefore, the detection of the DR in the early stages is highly recommended. However, a delay is registered for ensuring early DR diagnosis which caused by the low-rate of the ophthalmologists, the deficiency of diagnosis equipment and the lack of mobility of elderly patients.

In this paper, the main objective is to provide a mobile-aided screening system of moderate DR. Within this aim, we propose a classifier-based method which is based on detecting the Hard Exudate (HE) lesions that occur in moderate DR stage. A set of features are extracted to ensure an accurate and robust detection with respect to modest quality of fundus images. Moreover, the detection is provided in a low complexity processing to be suitable for mobile device. The aimed system corresponds to the implementation of the method on a smartphone associated to an optical lens for capturing fundus image. The system reached satisfactory screening performance where an accuracy of $98.36 \%$, a sensitivity of $100 \%$ and specificity of $96.45 \%$ are registered using the DIARETDB1 fundus image databases. Moreover, the screening is performed in an average execution time of 2.68 seconds.
\end{abstract}

Keywords: Fundus image; diabetic retinopathy; hard exudates; SVM; mobile-aided screening system.

\section{INTRODUCTION}

The Diabetic Retinopathy (DR) is an eye abnormality caused by long-term diabetes which it represents the leading cause of blindness of middle-aged and elderly people [1,29]. The DR prevalence is increasing at an alarming rate in world-wide where the number of DR-patient has been estimated to rise 126.6 million in 2030 [3]. The DR progresses through 4 stages what are mild, moderate, sever and proliferative DR [2]. In the mild stage, the micro-aneurysm lesions appear as small red dots caused by the dilation of thin vessel. This stage does not require referring to an ophthalmologist unless Diabetic Macular Edema is joined [40,41]. The moderate DR is deduced when Hard Exudate "HE" lesions [4] crop up, which are formed by lipids and proteins leaked from blood vessels. The HE appears as bright yellow clusters of varying shapes, sizes and locations. their sizes can vary from very few pixels to a large lesion as an Optic Disk (OD). Patients in moderate DRstage have a risk up to $27 \%$ to achieve proliferative stage within one year [40,41]. For that reason, the detection of the DR in the early stages is highly recommended.

However, a delay is registered for ensuring early DR diagnosis caused by: the low-rate of the ophthalmologists [24]; the deficiency of diagnosis equipment; and the lack of mobility of elderly patients. In order to solve those problems, many automatic detection methods were proposed for early DR diagnosis, particularly for HE detection that indicates at least reaching the moderate DR $[2,9,59,60,61,62]$. In the last few years, several optical lens have been put forward in order to capture the fundus images using the smartphone camera [30, 32, 35]. Those gadgets are well known with their low prices and the modest quality of captured images $[25,26]$. In fact, many works proved that ophthalmologists are able to predict the presence of the different DR lesions from smartphone captured fundus images. The clinical study described in [33] has shown a sensitivity of $95 \%$ and specificity of $94 \%$ when detecting the DR lesions on fundus images taken by mobile smartphone. In [34], the ophthalmologists were detected DR with $92.7 \%$ of sensitivity, $98.4 \%$ of specificity and 0.90 of 
Kappa (k) using the mobile phone (FOP) camera. Moreover, the D-eye lens was allowed distinguishing the HEs with a Kappa coefficient between 0.822 and 0.878 [31].

Therefore, the challenge is to propose an automated method for DR lesion detection that: (1) ensure a higher performance detection; (2) even with the moderate quality of smartphone captured fundus images; (3) and having a lower complexity to provide result in a reduced execution time. The implementation of the method on a smartphone associated with an optical lens for capturing fundus image relies to a mobile-aided screening system. The proposed system will be able to be used by non-ophthalmologist doctor to detect the DR. Based on it mobility and low price, such system presents an adequate solution to remedy the problems related to the delay on DR screening.

Within this objective, several state-of-the-art methods were proposed. The work described in [63] addressed the problems of limited field of view and quality of smartphone captured fundus images. Hence, an automated method was proposed to ensure a higher performance DR grading even with blurred classical fundus images. The execution time was evaluated only when method was run in desktop. Our first work described in [37] was aimed to provide DR grading of classical fundus images when an overall accuracy of $87.4 \%$ was achieved. The method was implemented in smartphone where classification takes between 200 and 250 milli-seconds. In [64], a method of HE detection is propounded where a trained model was stored on cloud platform. This method was performed a higher performance detection with an accuracy of $98 \%$ using a private classical image dataset, despite providing the decision into one minute and 15 seconds.

In this paper, we propose a novel automated method for moderate DR screening achieving a better trade-off between HE detection rate and the detection time. The method is based on classifier-based method for high performant locating of HE in fundus images. In addition, the processing has a low complexity to be suitable for mobile implementation. The proposed method is implemented into a smartphone to provide an accurate and fast mobile-aided screening system for moderate DR. Our paper is organized as follow. In section 2, we present the different processing steps of our method. In section 3, we describe the implementation of the proposed method to provide the mobile-aided screening system. The experimentation is depicted in section 4 where achieved results in terms of detection performance and execution time are detailed. The last section is dedicated to the discussion and the conclusion.

\section{THE PROPOSED METHOD FOR HARD EXUDATE DETECTION}

Having a fundus image as an input, the method pipeline is composed by three main processing. The first step consists in removing the $\mathrm{OD}$ and partitioning the retina into several candidate regions. Thereafter, a set of features are extracted for each region which reflect the HE characteristics. The third step brings to classify regions as clear or containing HE lesions.

\subsection{Pre-processing}

\subsubsection{Optic disk location}

The HEs are considered as bright regions in a way that their intensities are closed to the OD one. Moreover, the lipid leakage involves a yellow color similar to the color of optic nerve fibers. Therefore, this similarity leads to increase false positive shapes when detecting the HEs. Accordingly, it is essential to remove OD, as done in several HE detection methods $[12,16,9,5,11]$. The method proposed in [23] insures locating the OD based on circularity and intensity through applying the radon transform. The method achieved a higher accurate performance among the existing methods [13, 18], where an accuracy of $100 \%$ is performed to locate OD in the public DRIVE database. We note that this method is able to be configured with the aim of locating a sub-image having the same OD size. Thereafter, this method is extended to be provide a mobile computer aided system for OD detection [8]. The processing was optimized to reduce complexity where the same detection accuracy is performed through 1.3 second in smartphone device. Therefore, the method proposed by [8] will be implemented which extract the OD sub-image [18] in order to remove it from the fundus image.

\subsubsection{Fundus image partition}

Several methods have proceeded to browse all image pixels, which bring to false positive detection caused by noise pixels. Moreover, such processing leads to higher computational complexity methods. With respect to the objective of DR moderate screening, we proceed to assess the image by region instead by pixels, in order to rise the accuracy, the robustness and to enhance the computational efficiency. For such need, fundus image is partitioned into region based on retinal shapes in order to check the HE existence on each region separately.

The partition is ensured by applying the super-pixel algorithm $[39,57]$ which recognized by its feasibility, robustness and low complexity [42] where regions are defined based on color and shape borders. The LSC super-pixel algorithm spreads the fundus image while trying to minimize regions by providing them with nearest areas. Knowing that HEs may 
occur as small distributed lesions, this algorithm dispatches HE lesions into different regions, as illustrated in the fundus image of Fig.1 (a) which is partitioned into 462 regions. The SLIC super-pixel algorithm partitions iteratively the fundus image until all region pixels have similar morphologies. However, it involves muffled small HE lesions with relatively wide area, as indicated depicted in fundus image of Fig.1 (b) despite that it is partitioned into 612 regions. The SEEDS super-pixel algorithm provides better partitioning and succeeds to extract each HE cluster in a single region [55] without exceed 616 regions. Moreover, retinal background with stationary color and shape are extracted in single wide region, as shown in Fig.1 (c). Accordingly, we proceed to employ the SEEDS super-pixel algorithm.
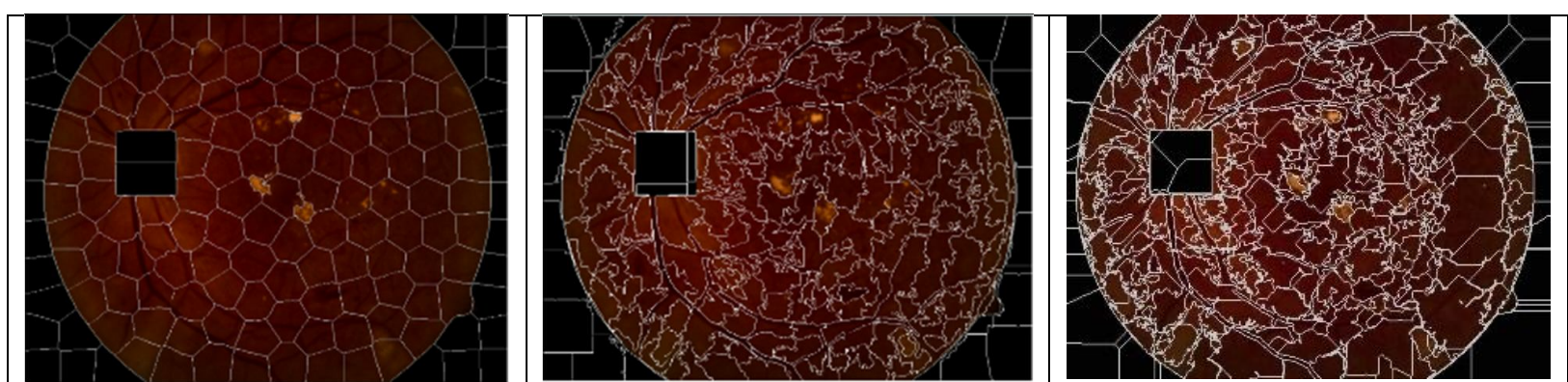

Figure 1. Super-pixel results: (a) the LSC algorithm; (b) the SLIC algorithm; (c) the SEEDS algorithm

\subsection{Feature extraction}

The HE lesions have several morphological proprieties where the main ones are the higher intensity, the yellow color and the sharp border. the proposed optical lenses allow light leakage when capturing fundus images. Therefore, it involves brightness noise shapes that prevents optimal detection of HE lesions. Elsewhere, the handheld aspect brings to provide a blurred fundus image which inhibits modelling explicitly the HE shapes. To surpass those limitations, we proceed to extract three features reflecting respectively intensity, color and sharp border, as described in the following sub-sections.

\subsubsection{Sharp border feature}

The HEs arise due to lipid leakage from blood vessel, which remain bundled in the retina. Therefore, the HE lesions are characterized by sharp borders, in contrast to the others DR lesions [15]. Accordingly, we proceed to generate a feature that reflexes the sharp edge of HEs. Among several edge detection methods [43], we employ the Sobel edge detector [44] which has a smoothing effect on the random noise in the image. The Sobel edge filter is applied by calculating a corresponding Gradient Magnitude (GM) of the fundus image to get the edge image as output [45], as described in eq.(1).

$$
G M(x, y)=|\vec{\nabla} I(x, y)|=\sqrt{G_{x}^{2}+G_{y}^{2}}
$$

Where $G_{x}$ and $G_{y}$ are the derivatives in horizontal and vertical directions, using respectively the mask $\left(\begin{array}{ccc}-1 & 0 & 1 \\ -2 & 0 & 2 \\ -1 & 0 & 1\end{array}\right)$ and the mask $\left(\begin{array}{ccc}-1 & -2 & -1 \\ 0 & 0 & 0 \\ 1 & 2 & 1\end{array}\right)$ to each pixel. Our method consists of providing a single feature that reflect the HE sharp edge. Within this objective, we compute the means edge value of each candidate region as indicated in eq.(2).

$$
M_{\text {Sobel }}=\frac{1}{n * m} \sum_{1}^{m} \sum_{1}^{n} G M(x, y)
$$

where $G M$ is the result of the sobel edge filter and $n * m$ is the sub-image size. The figure 2 (b) corresponds to the result of the sobel edge filter where the HE borders are illustrated explicitly modelled. Similarly, the retina background is depicted with lower intensities since it is characterized by a smoothed texture. Indeed, the region containing the HE lesions which are depicted with red borders incorporate the edges detected through the sobel filter, as shown in Fig.2 (f).

\subsubsection{Brightness feature}

The leaked of lipids and proteins derived from the retinal blood vessels [2] appear as bright yellow crystalline granules in the retinal image. Therefore, the HEs are considered as bright lesions having higher intensity values [20], compared to the other retinal components such as blood vessels, fovea, and red lesions such as haemorrhage and micro-aneurysm [46]. For that reason, we aim to extract a feature that reflects the brightness of HEs. In fact, HE lesions appear as "whiter" gray level than most of the rest of the image $[47,48,49]$. Therefore, we proceed to extract the gray-scale channel to model HE 
as brightness shapes in the retinal images, as shown in Fig.2 (c). Thereafter, the mean gray value of each candidate region is calculated as described in eq.(3).

$$
M_{\text {Gray }}=\frac{1}{n * m} \sum_{1}^{m} \sum_{1}^{n} I_{\text {Gray }}(x, y)
$$

Where $\mathrm{I}_{\mathrm{Gray}}$ is the gray-scale channel of the input fundus image and $n * m$ is the sub-image size.

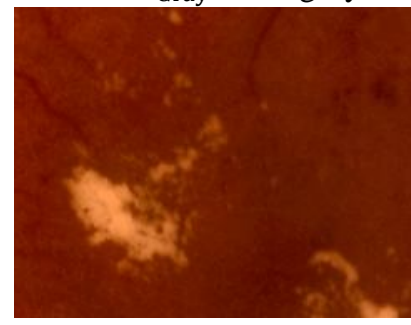

(a)

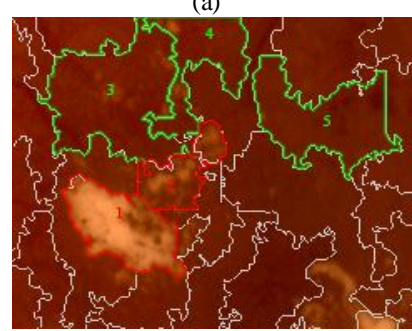

(e)

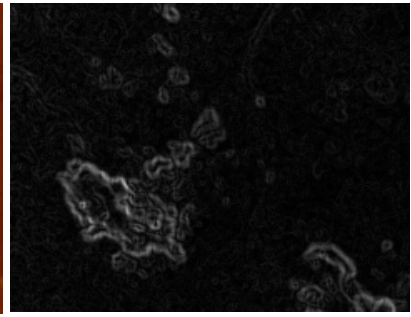

(b)

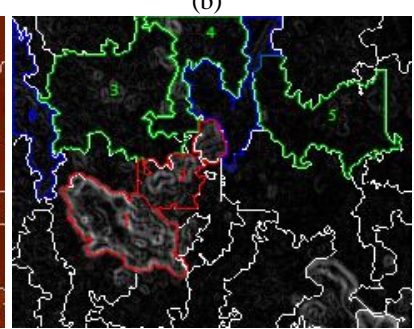

(f)

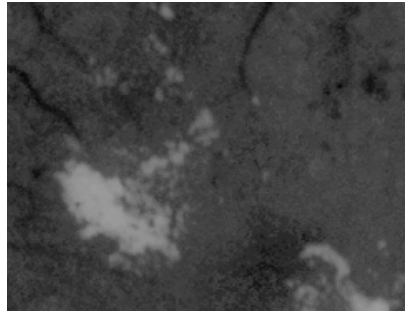

(c)

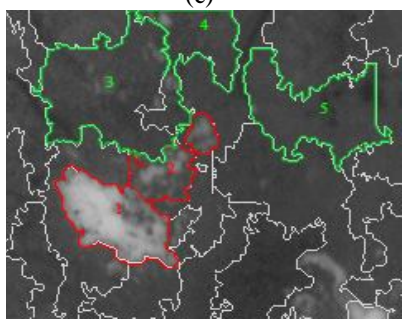

(g)

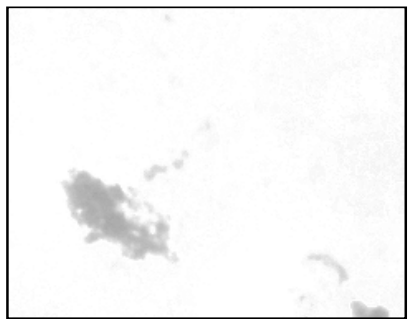

(d)

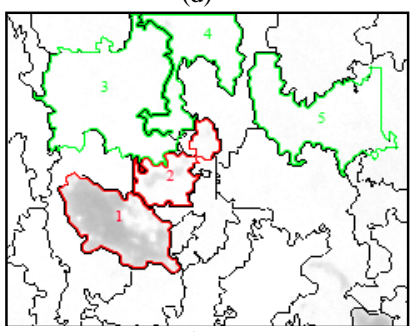

(h)

Figure 2. HE candidates in sub-image regions: (a) the original sub-image, (b) the sobel edge filter result; (c) the gray channel; (d) the yellow channel; (e-h) the result of applying the super-pixel algorithm of the sub-images (a-d)

\subsubsection{Color feature}

The HE lesions spread upward the retina with dense composition. Therefore, there are characterized by yellowish color without a red shade, contrary to the retina background, the vessel tree and the others DR lesions. Moreover, the HE color remain the same whatever the HE sizes, locations and shapes [19][50]. For that reason, we aim to extract a feature that reflects the color of HE. For this purpose, the fundus image is converted from RGB space to the HSV color space in order to select the Hue channels which is defined as the ratio of the red and the green value of each pixel[51]. Consequently, the HE lesions appear as dark shapes in relation to the other retinal components, such has illustrated in Fig.2 (e). Thereafter, our method consists in computing the means value of the color features of each candidate region as expressed in eq.(4).

$$
M_{\text {Color }}=\frac{1}{n * m} \sum_{1}^{m} \sum_{1}^{n} I_{\text {Color }}(x, y)
$$

Where $I_{\text {color }}$ is the hue image and $n * m$ is the sub-image size.

\subsection{Classification}

In this section, the objective is to choose a classifier that ensures a high performance classification through the extracted features. In addition, the training should be ensured with a small dataset size due to the lack a smartphone captured fundus image database. Besides, the classifier should have a lower complexity to provide result on a reduced execution time. Within this Objective, we select three classifiers that are the Support Vector Machine (SVM), the Random Forest (RF) and the K-Nearest Neighbors (KNN). The result of each classifier depend on how the parameters are initialized. For that, each classifier is experimentally employed with different values of parameters, as described in section 4.1.2. Hence, the classifier that achieves better performances is selected to be employed on our method.

\section{MOBILE-AIDED SCREENING SYSTEM OF MODERATE DIABETIC RETINOPATHY}

The method is implemented as an app in an android mobile device, using the android studio editor where machine learning and image processing are performed through the Open computer vision (OpenCV) library [36, 52]. This library contains over than 2500 optimized functions which is used in many areas such as medical imaging and security. In addition, the OpenCV library also includes a general-purpose Machine Learning Library (MLL)[52]. To create our Android app, an access to the operating system components is needed which is assured by a high-level Application Programming Interface (API) available in JAVA language [47]. The method is implemented in the application layer as indicated in Fig.3. The functions related to the image processing, training and testing the classifier were called through the JAVA Native Interface 
(JNI) combined with the OpenCV library. The access to the OpenCV library could be done either using a direct JNI call or through Java API following, as modelled by arrows in Fig.3.

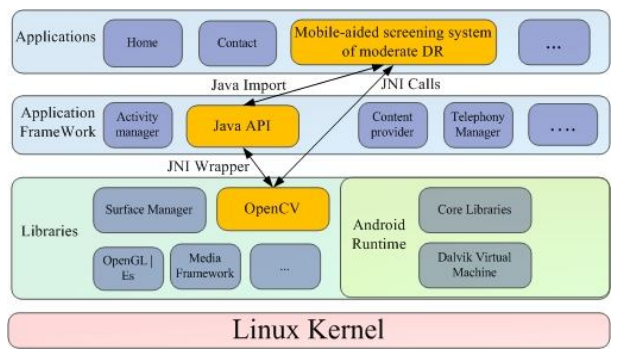

Figure 3. Illustration of the connection between the developed application, the Java API and the OpenCV library

The developed user interface of the mobile app is shown in Fig.4, which contain buttons for loading and testing a fundus image. Similarly, the HE detection result and the execution time are illustrated are shown above the selected image.

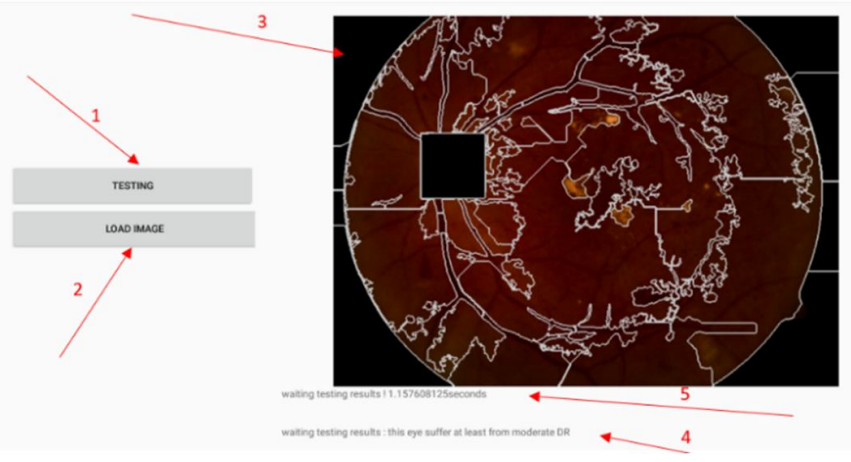

Figure 4. The user interface of the mobile-aided screening system

\section{EXPERIMENTAL RESULTS}

\subsection{Evaluation of the HE detection method}

\subsubsection{Databases and evaluation metrics}

For the evaluation experiment, the DiaretDB1 database is selected which is commonly used by automated methods for DR screening, where some fundus images are illustrated in Fig 5. It contains 89 fundus images having a size of $1500 \times$ 1152 captured by digital camera with a 50-degree field of view. The HE lesions have been manually located by four independent medical experts. The performance of our application will be measured based on image-based criterion: if fundus image contains at least one HE regions, this image is considered "at least in moderate DR stage". Otherwise, it considered as "healthy or in mild DR stage" $[21,22]$. We aim to evaluate the ability of our method to identify correctly each class. Within this objective, the performance will be measured through the accuracy, the sensitivity and the specificity metrics which are computed as described eq.(5), eq.(6) and eq.(7).

$$
\begin{aligned}
& \text { Accuracy }=\frac{T P+T N}{(T P+T N+F P+F N)} \\
& \text { Sensitivity }=\frac{T P}{(T P+F N)} \\
& \text { Specificity }=\frac{T N}{(T N+F P)}
\end{aligned}
$$

Where TP (True Positive) is the number of the abnormal images correctly classified, FP (False Positive) is the number of normal images wrongly classifier as abnormal, TN (True Negative) normal images correctly classified as healthy and FN (False Negative) is the number of abnormal images wrongly classified as normal.

\subsubsection{Performance evaluation of the HE detection method}

The goal of our method is to detect the HE presence on regions, separately. With this aim, the superpixel algorithm is applied to the fundus images, where 160 regions are selected from each database to perform the evaluation. Thereafter, the feature set is extracted for each region. Thereafter, the three classifiers are trained using the input features. the SVM is 
tested separately with the sigmoid, the polynomial and the RBF kernels, while the $\mathrm{C}, \gamma$ and d parameters are fixed using the grid search method [54]. For the RF classifier, the forest trees are tested with 6,8 and 10 depths while feature number and tree are fixed to three and 500. The KNN classifier is trained where cluster size $\mathrm{K}$ is varied following the above values $\{5,7,9\}$. The performance metrics of each classifier is show in Table 1 , where we deduce that using the SVM with the polynomial kernel allows achieving the highest detection performance with an accuracy of $98.36 \%$, a sensitivity of $100 \%$ and a specificity of $96.45 \%$. In addition, we deduce that the detection performance remains highly, despite of the different classifier and their parameter, which attests that extracted features reflect the HE criteria.

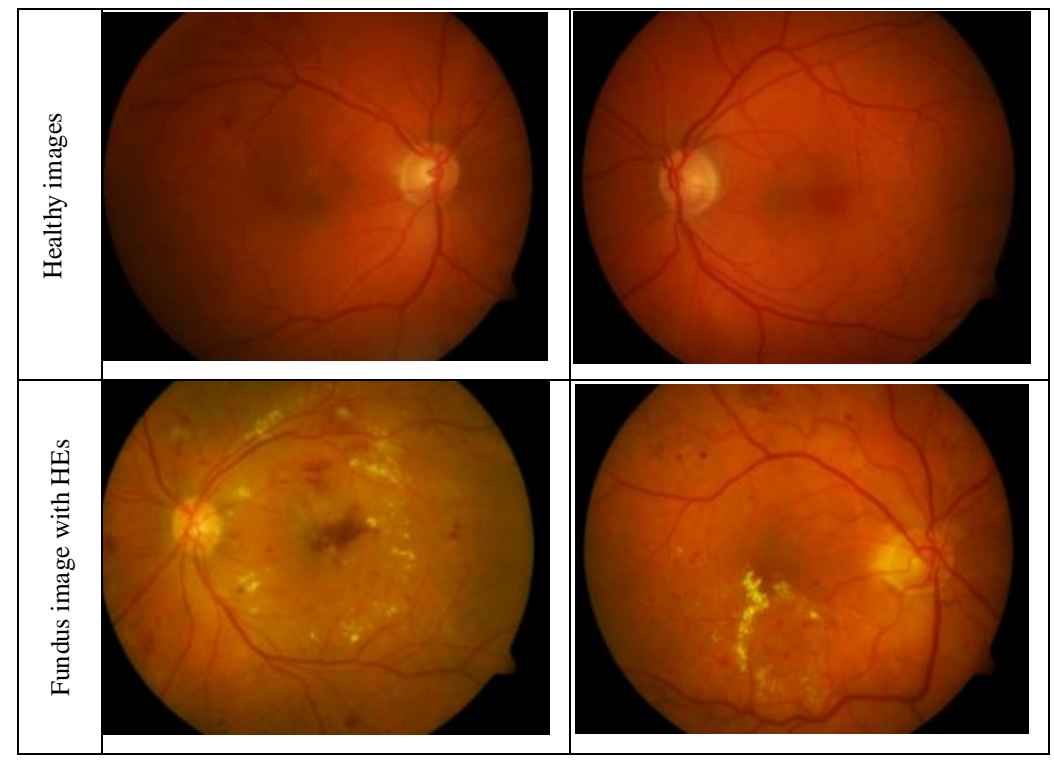

Figure 5. healthy and DR-affected fundus images from DIARETDB 1 database

Table 1. HE Detection performance in terms of classifiers and classifier parameters

\begin{tabular}{|c|c|c|c|c|c|c|c|c|c|}
\hline Classifier & \multicolumn{3}{|c|}{ SVM } & \multicolumn{3}{c|}{ RF } & \multicolumn{3}{c|}{ KNN } \\
\hline & Sigmoid & RBF & Poly & depth $=6$ & 8 & 10 & 5 & 7 & 9 \\
\hline Acc (\%) & 88.57 & 97.95 & $\mathbf{9 8 . 3 6}$ & 97.75 & 97.55 & 96.53 & 95.91 & 93.87 & 95.91 \\
\hline Sens (\%) & 86.82 & 98.32 & $\mathbf{1 0 0}$ & 97.16 & 96.50 & 96.42 & 100 & 96.15 & 100 \\
\hline Spec (\%) & 90.92 & 97.67 & $\mathbf{9 6 . 4 5}$ & 98.12 & 98.46 & 96.39 & 92 & 91.30 & 92 \\
\hline
\end{tabular}

\subsubsection{Evaluation with respect to existing methods}

To ensure a fair evaluation, the provided performance metrics are compared to the ones achieved be existing methods using the same DiaretDB1 dataset, as depicted in Table 2. Our method outperforms the detection performance of the methods proposed in [2, 9, 61, 62]. It achieves a higher accuracy than the ones recently proposed in [2, 59, 61, 62]. Moreover, our method registers the highest sensitivity compared to all methods. This result is caused by the adequate choice of the HE properties and the corresponding features. Accordingly, the achieved performance is promising for diagnosis through smartphone captured images.

Table 2. Comparative results for image-based evaluation for the proposed method

\begin{tabular}{cccc}
\hline Method & Accuracy & Sensitivity & Specificity \\
\hline B. Harangi 2014 [62] & 82 & 86 & -- \\
\hline M. Fraz 2017 [61] & 87 & 92 & 81 \\
\hline W. Zhou 2017 [9] & --- & 87.56 & 94.65 \\
\hline J. Kaur 2018 [2] & $87 \pm 4.29$ & $91 \pm 3.34$ & $94 \pm 2.28$ \\
\hline K. Adem 2018 [60] & 98.53 & 99.2 & 97.97 \\
\hline S. Long 2019 [59] & 97.7 & 97.5 & 97.8 \\
\hline Proposed method & $\mathbf{9 8 . 3 6}$ & $\mathbf{1 0 0}$ & $\mathbf{9 6 . 4 5}$ \\
\hline
\end{tabular}




\subsection{Evaluation of the mobile CAD system for HE detection}

\subsubsection{Complexity analysis of the proposed method}

The OD location method described in [8] consists at applying the radon transform to a fundus image with a size of $(n \times$ $n$ ) in order to model intensity. Thereafter, a radon space is extracted for each sub-image in order to locate OD based on pic of intensities. Therefore, the OD location requires $O\left(n^{2}\right)$ to be performed. The SEEDS superpixel algorithm leads to partition fundus image into regions and refine iteratively the regions boundary, which have a complexity of $O(n$. $\log (n))$ [27]. Each feature leads to explore pixels of each region to provide the feature value. Knowing that the whole image is partitioned into regions, each feature and hence the whole feature extraction step requires $O\left(n^{2}\right)$ de be done. The SVM testing consists at injecting features in the trained model to predict the classification [28]. Therefore, a constant time complexity equal to $O(1)$ is required. Accordingly, the whole proposed method for HE detection has a complexity of $O\left(n^{2}\right)$.

\subsubsection{The execution time evaluation}

The implementation is run into the smartphone "OnePlus 5" where hardware and software parameters are detailed in Table 3.

Table

Table 3. The technical sheets of the "OnePlus 5" Smartphone

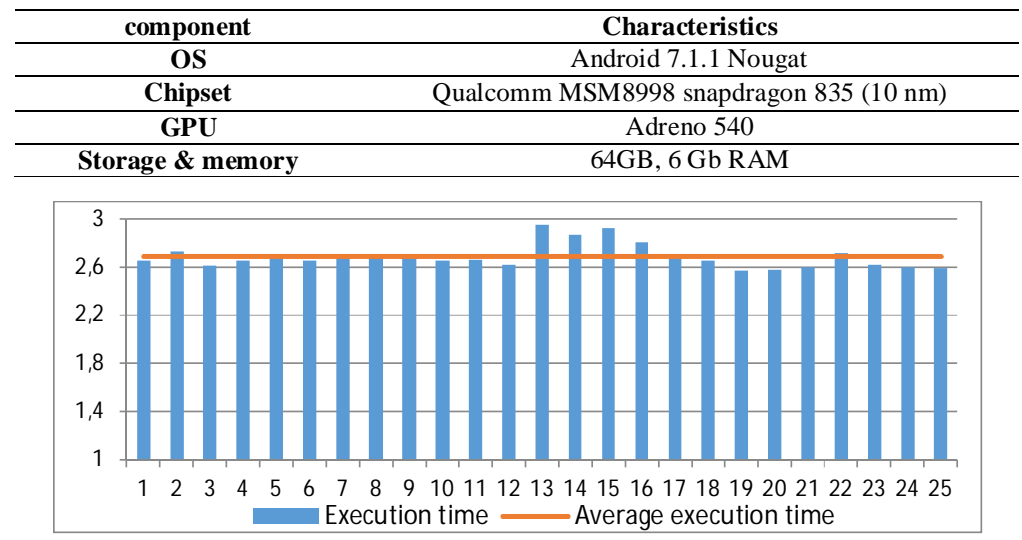

Figure 6. execution time of the mobile-aided screening system for 25 EyePACS fundus images

Therefore, the execution time of our implementation is compared to state-of-the art methods where their computational performances are shown in Table 4. Aside the method proposed in [64], we note that the indicated methods are always implemented and executed using MATLAB software tool in desktop architectures which have wide powerful processing compared to the mobile device. Despite, our method involves the fastest HE detection compared to the indicated works.

Table 4. Comparison of execution time with existing HE detection methods

\begin{tabular}{cc}
\hline HE detection methods & Execution time (in seconds) \\
\hline L. Giancardo 2012[10] & 9.3 \\
\hline X. Zhang 2014[7] & 35 \\
\hline I. N. Figueiredo 2015[17] & 15.97 \\
\hline A. R. G. Harwood 2017 [47] & 240 \\
\hline J. H. Tan 2017[1] & From 3,4 to 4,1 \\
\hline W. Zhou 2017 [9] & $10 \sim 19$ \\
\hline J. Kaur 2018 [2] & 9.36 \\
\hline E. Saeed [64] & 75 \\
\hline Proposed method & $\mathbf{2 . 6 8}$
\end{tabular}

\section{CONCLUSION}

The (DR) requires to be diagnosed in early stages. The lack of ophthalmologist and imaging equipment cause a delay on early DR diagnosis, and hence contribute to the wide number of patients having damaged vision caused by DR. In this paper, we have proposed a mobile-aided screening system for moderate DR based on detecting the HE lesions. The experimental results allow to deduce a satisfactory performance of DR screening and prove the robustness of the method 
against modest quality of fundus images. Moreover, the low complexity of the method processing involves performing screening in reduced execution time which is suitable for clinical context.

In near future, we aim to enhance performance of the proposed system where HE location can be improved taking into account their neighborhood of blood vessel tree and DR red lesions. the preprocessing can also be extended to rectify blur and noise that occur due to the handheld aspect of smartphone capturing. Moreover, the system can be extended to provide a DR classification whatever the stage is, through detection all DR lesions. The classifier is able to be replace by a deep learning architecture, as the one proposed in [37]. Elsewhere, the timing performance can be improved by taking benefit from the parallelism offered by recent smartphone architectures.

\section{REFERENCES}

[1] J. H. Tan et al., 'Automated segmentation of exudates, haemorrhages, microaneurysms using single convolutional neural network', Inf. Sci., vol. 420, pp. 66-76, Dec. 2017.

[2] J. Kaur and D. Mittal, 'A generalized method for the segmentation of exudates from pathological retinal fundus images', Biocybern. Biomed. Eng., vol. 38, no. 1, pp. 27-53, Jan. 2018.

[3] Y. Zheng, M. He, and N. Congdon, 'The worldwide epidemic of diabetic retinopathy', Indian J. Ophthal., vol. 60, no. 5, pp. 428-431, 2012.

[4] J. Amin, M. Sharif, M. Yasmin, H. Ali, and S. L. Fernandes, 'A method for the detection and classification of diabetic retinopathy using structural predictors of bright lesions', J. Comput. Sci., vol. 19, pp. 153-164, Mar. 2017.

[5] W. Kusakunniran, Q. Wu, P. Ritthipravat, and J. Zhang, 'Hard exudates segmentation based on learned initial seeds and iterative graph cut', Comput. Methods Programs Biomed., vol. 158, pp. 173-183, May 2018.

[6] P. Prentašić and S. Lončarić, 'Detection of exudates in fundus photographs using deep neural networks and anatomical landmark detection fusion', Comput. Methods Programs Biomed., vol. 137, pp. 281-292, Dec. 2016.

[7] X. Zhang et al., 'Exudate detection in color retinal images for mass screening of diabetic retinopathy', Med. Image Anal., vol. 18, no. 7, pp. 1026-1043, Oct. 2014.

[8] ELLOUMI Y, AKIL M, KEHTARNAVAZ N. mobile computer aided system for optic nerve head detection.Computer Methods and Programs in Biomedicine (CMPB).August 2018.Vol. 162, pages 139-148.

[9] W. Zhou, C. Wu, Y. Yi, and W. Du, 'Automatic Detection of Exudates in Digital Color Fundus Images Using Superpixel Multi-Feature Classification', IEEE Access, vol. 5, pp. 17077-17088, 2017.

[10] L. Giancardo et al., 'Exudate-based diabetic macular edema detection in fundus images using publicly available datasets', Med. Image Anal., vol. 16, no. 1, pp. 216-226, Jan. 2012.

[11] K. Wisaeng and W. Sa-ngiamvibool, 'Improved fuzzy C-means clustering in the process of exudates detection using mathematical morphology', Soft Comput., vol. 22, no. 8, pp. 2753-2764, Apr. 2018.

[12] S. Banerjee and D. Kayal, 'Detection of hard exudates using mean shift and normalized cut method', Biocybern. Biomed. Eng., vol. 36, no. 4, pp. 679-685, Jan. 2016.

[13] Haleem M. S, Han L, van Hemert J, and Li B.Automatic extraction of retinal features from colour retinal images for glaucoma diagnosis: a review.Comput Med Imaging Graph. Dec. 2013;vol. 37, no. 7-8, pp. 581-596.

[14] E. Imani and H.-R. Pourreza, 'A novel method for retinal exudate segmentation using signal separation algorithm', Comput. Methods Programs Biomed., vol. 133, pp. 195-205, Sep. 2016.

[15] C. I. Sánchez, M. García, A. Mayo, M. I. López, and R. Hornero, 'Retinal image analysis based on mixture models to detect hard exudates', Med. Image Anal., vol. 13, no. 4, pp. 650-658, Aug. 2009.

[16] D. Kayal and S. Banerjee, 'Detection of hard exudates using 2D Otsu algorithm in digital retinal fundus image', CSI Trans. ICT, vol. 5, no. 1, pp. 53-57, Mar. 2017.

[17] I. N. Figueiredo, S. Kumar, C. M. Oliveira, J. D. Ramos, and B. Engquist, 'Automated lesion detectors in retinal fundus images', Comput. Biol. Med., vol. 66, pp. 47-65, Nov. 2015.

[18] S. BEN SAYADIA, Y. ELLOUMI, M. AKIL, M. H. BEDOUI, "Computational Efficiency of Optic Disk Detection on Fundus Image: A survey", SPIE Proceeding on Real-Time Image and Video Processing 2018, May 2018, Orlando, Florida (United States).

[19] G. Fang, N. Yang, H. Lu, and K. Li, 'Automatic segmentation of hard exudates in fundus images based on boosted soft segmentation', in 2010 International Conference on Intelligent Control and Information Processing, 2010, pp. 633-638.

[20] S. Joshi and P. T. Karule, 'A review on exudates detection methods for diabetic retinopathy', Biomed. Pharmacother. Biomedecine Pharmacother., vol. 97, pp. 1454-1460, Jan. 2018. 
[21] H. Jelinek and M. J. Cree, Automated Image Detection of Retinal Pathology, 1 edition. Boca Raton: CRC Press, 2009.

[22] A. Osareh, B. Shadgar, and R. Markham, 'A Computational-Intelligence-Based Approach for Detection of Exudates in Diabetic Retinopathy Images’, IEEE Trans. Inf. Technol. Biomed., vol. 13, no. 4, pp. 535-545, Jul. 2009.

[23] Shahri, R.P., M. Tavakoli, N. Kehtarnavaz, 2014."Computationally efficient optic nerve head detection in retinal fundus images”, Biomedical Signal Processing and Control, vol. 11,pp. 63-73.

[24] S. Resnikoff, W. Felch, T.-M. Gauthier, and B. Spivey, 'The number of ophthalmologists in practice and training worldwide: a growing gap despite more than 200,000 practitioners', J. Ophthalmol., vol. 96, no. 6, pp. 783-787, 2012.

[25] J. M. Micheletti, A. M. Hendrick, F. N. Khan, D. C. Ziemer, and F. J. Pasquel, 'Current and Next Generation Portable Screening Devices for Diabetic Retinopathy', J. Diabetes Sci. Technol., vol. 10, no. 2, pp. 295-300, Feb. 2016.

[26] S. Darma, F. Zantvoord, and F. D. Verbraak, 'The quality and usability of smartphone and hand-held fundus photography, compared to standard fundus photography', Acta Ophthalmol. (Copenh.), vol. 93, no. 4, pp. e310-311, Jun. 2015.

[27] Yongxia Zhang, Xuemei Li, Xifeng Gao, and Caiming Zhang, "A Simple Algorithm of Superpixel Segmentation With Boundary Constraint", IEEE TRANSACTIONS ON CIRCUITS AND SYSTEMS FOR VIDEO TECHNOLOGY, VOL. 27, NO. 7, JULY 2017.

[28] Ayinala, M., Parhi, K.K.: Low complexity algorithm for seizure prediction using Adaboost. In: 2012 Annual International Conference of the IEEE Engineering in Medicine and Biology Society. pp. 1061-1064 (2012)

[29] A. Bastawrous and B. D. Hennig, 'The global inverse care law: a distorted map of blindness', Br. J. Ophtha., vol.96, no.10, pp.1357-1358, Oct. 2012.

[30] N. Panwar et al., 'Fundus Photography in the 21st Century--A Review of Recent Technological Advances and Their Implications for Worldwide Healthcare', Telemed. J. E-Health Off. J. Am. Telemed. Assoc., vol. 22, no. 3, pp. 198208, Mar. 2016.

[31] M. L. Muiesan et al., 'Ocular fundus photography with a smartphone device in acute hypertension', J. Hypertens., vol. 35 , no. 8, pp. 1660-1665, 2017.

[32] N. M. Bolster, M. E. Giardini, I. A. Livingstone, and A. Bastawrous, 'How the smartphone is driving the eye-health imaging revolution', Expert Rev. Ophthalmol., vol. 9, no. 6, pp. 475-485, Dec. 2014.

[33] M. E. Ryan et al., 'Comparison Among Methods of Retinopathy Assessment (CAMRA) Study: Smartphone, Nonmydriatic, and Mydriatic Photography', Ophthalmology, vol. 122, no. 10, pp. 2038-2043, Oct. 2015.

[34] R. Rajalakshmi et al., 'Validation of Smartphone Based Retinal Photography for Diabetic Retinopathy Screening', PLoS ONE, vol. 10, no. 9, Sep. 2015.

[35] A. Russo et al., 'Comparison of Smartphone Ophthalmoscopy With Slit-Lamp Biomicroscopy for Grading Vertical Cup-to-Disc Ratio', J. Glaucoma, vol. 25, no. 9, pp. e777-781, 2016.

[36] M. A. Hudelist, C. Cobârzan, and K. Schoeffmann, 'OpenCV Performance Measurements on Mobile Devices', in Proceedings of International Conference on Multimedia Retrieval, New York, NY, USA, 2014, pp. 479:479-479:482.

[37] S. MAJUMDER, Y. ELLOUMI, M. AKIL, R. KACHOURI, N. KEHTARNAVAZ, "A deep learning-based smartphone app for real-time detection of five stages of Diabetic Retinopathy", Real-Time Image Processing and Deep Learning 2020, 27 - 28 April 2020.

[38] C. I. Sánchez, R. Hornero, M. I. López, M. Aboy, J. Poza, and D. Abásolo, 'A novel automatic image processing algorithm for detection of hard exudates based on retinal image analysis', Med. Eng. Phys., vol. 30, no. 3, pp. 350357, Apr. 2008.

[39] M. Van den Bergh, X. Boix, G. Roig, and L. Van Gool, 'SEEDS: Superpixels Extracted Via Energy-Driven Sampling', Int. J. Comput. Vis., vol. 111, no. 3, pp. 298-314, Feb. 2015.

[40] American Optometric Association. Evidence-based Clinical Practice Guideline.

[41] Grading diabetic retinopathy from stereoscopic color fundus photographs — an extension of the modified Airlie House classification: ETDRS report number 10. Early Treatment Diabetic Retinopathy Study Research roup. Ophthalmology. 1991;98(5):786-806.

[42] L. Zhengqin and C. Jiansheng, 'Superpixel segmentation using Linear Spectral Clustering', in 2015 IEEE Conference on Computer Vision and Pattern Recognition (CVPR), 2015, pp. 1356-1363.

[43] S. Gupta and S. Mazumdar, 'Sobel Edge Detection Algorithm', 2013.

[44] V. Vijayakumari and N. Suriyanarayanan, 'Exudates Detection Methods in Retinal Images Using Image Processing Techniques', vol. 1, no. 2, p. 6, 2010.

[45] O. Vincent and O. Folorunso, 'A Descriptive Algorithm for Sobel Image Edge Detection', 2009.

[46] W. Zhou, C. Wu, D. Chen, Z. Wang, Y. Yi, and W. Du, 'Automated Detection of Red Lesions Using Superpixel Multichannel Multifeature', Computational and Mathematical Methods in Medicine, 2017. 
[47] A. R. G. Harwood and A. J. Revell, 'Parallelisation of an interactive lattice-Boltzmann method on an Androidpowered mobile device', Adv. Eng. Softw., vol. 104, pp. 38-50, Feb. 2017.

[48] S. Lahmiri and M. Boukadoum, 'Automated detection of circinate exudates in retina digital images using empirical mode decomposition and the entropy and uniformity of the intrinsic mode functions', Biomed. Tech. (Berl), vol. 59, no. 4, pp. 357-366, Aug. 2014.

[49] M. García, C. I. Sánchez, J. Poza, M. I. López, and R. Hornero, 'Detection of Hard Exudates in Retinal Images Using a Radial Basis Function Classifier', Ann. Biomed. Eng., vol. 37, no. 7, pp. 1448-1463, Jul. 2009.

[50] S. Rajan, T. Das, and R. Krishnakumar, 'An Analytical Method for the Detection of Exudates in Retinal Images Using Invertible Orientation Scores', 2016.

[51] D. E, R. V, and J. Pa, 'Identification of hard exudates in retinal images', Biomed. Res., vol. 0, no. 0, 2017.

[52] G. Bradski and A. Kaehler, Learning OpenCV: Computer Vision with OpenCV Library, ed. O'Reilly Media, 2008.

[53] V. Gulshan et al., 'Development and Validation of a Deep Learning Algorithm for Detection of Diabetic Retinopathy in Retinal Fundus Photographs', JAMA, vol. 316, no. 22, pp. 2402-2410, 132016.

[54] V. Vapnik, The Nature of Statistical Learning Theory, 2nd ed. New York: Springer-Verlag, 2000.

[55] M. V. den Bergh, D. Carton, and L. V. Gool, 'Depth SEEDS: Recovering incomplete depth data using superpixels', in 2013 IEEE Workshop on Applications of Computer Vision (WACV), 2013, pp. 363-368.

[56] Lili Xu and Shuqian Luo, 'Support vector machine based method for identifying hard exudates in retinal images', in Computing and Telecommunication 2009 IEEE Youth Conference on Information, 2009, pp. 138-141.

[57] D. Stutz, A. Hermans, and B. Leibe, 'Superpixels: An Evaluation of the State-of-the-Art', Comput. Vis. Image Underst., vol. 166, pp. 1-27, Jan. 2018.

[58] K. Tbarki, S. B. Said, R. Ksantini, and Z. Lachiri, 'RBF kernel based SVM classification for landmine detection and discrimination', in 2016 International Image Processing, Applications and Systems (IPAS), 2016, pp. 1-6.

[59] Shengchun Long, Xiaoxiao Huang, Zhiqing Chen, Shahina Pardhan, and Dingchang Zheng, "Automatic Detection of Hard Exudates in Color Retinal Images Using Dynamic Threshold and SVM Classification: Algorithm Development and Evaluation", BioMed Research International Volume 2019, Article ID 3926930, 13 pages.

[60] Kemal Adem, "Exudate detection for diabetic retinopathy with circular Hough transformation and convolutional neural networks", Expert Systems with Applications, Volume 114, 30 December 2018, Pages 289-295.

[61] M.M. Fraz, W. Jahangir, S. Zahid, M.M. Hamayun, S.A. Barman, Multiscale segmentation of exudates in retinal images using contextual cues and ensemble classification, Biomed. Signal Process. Control 35 (2017) 50-62.

[62] B. Harangi, A. Hajdu, Automatic exudate detection by fusing multiple active contours and regionwise classification, Comput. Biol. Med. 54 (2014) 156-171.

[63] Recep E.Hacisoftaoglu, MahmutKarakaya, Ahmed B.Sallam, "Deep Learning Frameworks for Diabetic Retinopathy Detection with Smartphone-based Retinal Imaging Systems", Pattern Recognition Letters, Volume 135, July 2020, Pages 409-417.

[64] Emil Saeed, Maciej Szymkowski, Khalid Saeed, and Zofia Mariak, "An Approach to Automatic Hard Exudate Detection in Retina Color Images by a Telemedicine System Based on the d-Eye Sensor and Image Processing Algorithms", Sensors (Basel). 2019 Feb; 19(3): 695.

Authors' background

\begin{tabular}{|l|l|l|l|}
\hline \multicolumn{1}{|c|}{ Your Name } & \multicolumn{1}{|c|}{ Title* } & \multicolumn{1}{c|}{ Research Field } & \multicolumn{1}{c|}{ Personal website } \\
\hline Yaroub ELLOUMI & $\begin{array}{l}\text { Assistant } \\
\text { professor }\end{array}$ & Computer Vision & https://sites.google.com/site/yaroubelloumi/ \\
\hline Manef BEN MBAREK & Phd candidate & Mobile Computing & --- \\
\hline Rahma BOUKADIDA & Phd candidate & Artificial Intelligence & --- \\
\hline Mohamed AKIL & Full professor & Real-Time Image Processing & https://cv.archives-ouvertes.fr/mohamed-akil \\
\hline Mohamed Hedi BEDOUI & Full professor & Biomedical Engineering & http://www.labtim.org/membre.php?id=LTIMJG8MF9 \\
\hline
\end{tabular}

